\title{
Correlation Analysis of PTPN22 Gene Polymorphism, Th17 I Treg Cells and Their Cytokines in GD with Different lodine Nutritional Status
}

\author{
Hou Zhenjiang ${ }^{1}{ }^{*}$, Mu Zhaoxin ${ }^{1}$, Wang Cuicui ${ }^{2}$, Feng Jing ${ }^{3}$, Chen Yunxia ${ }^{4}$, Ma Jinqun ${ }^{4}$, \\ Liu Chunyan ${ }^{4}$ \\ ${ }^{1}$ Cangzhou Thyroid Disease Engineering Technology Research Center, Cangzhou Medical College, Cangzhou, China \\ ${ }^{2}$ Department of Medical Technology, Cangzhou Medical College, Cangzhou, China \\ ${ }^{3}$ Cangzhou Central Hospital, Cangzhou, China \\ ${ }^{4}$ Endocrinology Department, Cangzhou People's Hospital, Cangzhou, China
}

Email address:

houzhenjiang@sina.com (Hou Zhenjiang)

${ }^{*}$ Corresponding author

\section{To cite this article:}

Hou Zhenjiang, Mu Zhaoxin, Wang Cuicui, Feng Jing, Chen Yunxia, Ma Jinqun, Liu Chunyan. Correlation Analysis of PTPN22 Gene Polymorphism, Th17 / Treg Cells and Their Cytokines in GD with Different Iodine Nutritional Status. Science Journal of Public Health. Vol. 9, No. 3, 2021, pp. 83-91. doi: 10.11648/j.sjph.20210903.13

Received: April 6, 2021; Accepted: May 10, 2021; Published: May 24, 2021

\begin{abstract}
Background: PTPN22 is an important candidate gene for autoimmune diseases and its SNP is involved in the pathogenesis of a variety of autoimmune diseases. Objective: To investigate the relationship between the PTPN22 gene polymorphism (rs2488457) and the Th17 and Treg cells and factors in the peripheral blood mononuclear cells (PBMC) of Graves' disease (GD) patients with different iodine nutrition conditions. Methods: We selected 100 GD patients and 60 healthy people in Cangzhou Central Hospital and People's Hospital from September 2019 to August 2020 and used SASP-PCR technology to calculate the PTPN22-1123 genotype and allele frequency. Flow cytometrywere used to detect the ratio and ratio of Th17 and Treg cells in PBMC and real-time PCR were used to detect the mRNA expression levels of ROR- $\gamma \mathrm{t}$ and Foxp3. ELISA method was used to detect serum IL-17 and TGF- $\beta$ levels. Results: The PTPN22 gene $-1123 \mathrm{G}>\mathrm{C}$ (rs2488457) of GD patients has a certain correlation with Th17 and Treg cells and their factors in PBMC. The distribution frequencies of various genotypes and alleles were statistically different between GD patients and the control group $(\mathrm{P}<0.05)$, but they had nothing to do with iodine nutrition status. The $\mathrm{C} / \mathrm{G}$ and $\mathrm{C} / \mathrm{C}$ genotypes at the PTPN22 gene $(1123 \mathrm{G}>\mathrm{C})$ of may increase the risk of GD, while the G/G genotype may reduce the risk of GD. Conclusion: PTPN22 gene polymorphism (rs2488457) in GD patients and Th17 and Treg cells and their factors in PBMC are related to GD susceptibility.
\end{abstract}

Keywords: PTPN22, Gene Polymorphism, Th17/Treg Cells and Factors, Iodine Nutritional Status, Graves' Disease

\section{Introduction}

Autoimmune thyroid diseases (AITD) are the most common thyroid diseases other than iodine deficiency diseases, and they are also common organ-specific autoimmune diseases in humans, and their incidence has been on the rise in recent years. AITD mainly includes Graves' Disease (GD disease) and Hashimoto thyroiditis (HT), with similar genetic and immunological basis. Prolonged exposure to environmental triggers can promote disease in people with specific genetic backgrounds. GD is a common clinical endocrine disease. It has the highest incidence among all types of hyperthyroidism, accounting for about $85 \%$ of the causes of hyperthyroidism [1]. The prevalence of GD in Western countries is about $0.5 \%$ to $2 \%$, and the prevalence in China is about $2 \%$ to $3.0 \%$ [2]. There are many causes of GD. Environmental, genetic and autoimmune dysfunction can all cause specific autoimmune reactions in the thyroid gland, producing thyroid peroxidase antibody (TPOAb), thyroglobulin antibody (TgAb) and other autoantibodies in the body. At the same time, it is accompanied by diffuse goiter, 
hyperthyroidism and hypermetabolic state. GD can occur at any age, but it is more common in women. Studies have found that there are many reasons for the increase in the incidence of GD, and iodine nutritional status may be one of the influencing factors. Excessive iodine intake increases the incidence of GD and the positive rate of thyroid autoantibodies, and as the disease worsens, the recurrence rate also increases [3]. The 10-year changes before and after Universal Salt iodization (USI) indicate that the incidence of AITD increases with the increase of iodine nutrition level. A large number of epidemiological investigations have been carried out on the relationship between urinary iodine (UI) and hyper-thyroidism and GD, but the results of the research are not the same. Recent studies have found that the main cytokines and specific transcription factors secreted by Th17 and Treg cells (such as IL-17, TGF- $\beta$, ROR- $\gamma$ t, Foxp3) play an important role in immune-suppression. It has become the current research direction of the mechanism of occurrence, development and outcome of immune-related diseases. In recent years, studies on Th17/Treg cells, ROR- $\gamma \mathrm{t}$ and Foxp 3 in RA and other AIDs have been widely carried out abroad [4], In recent years, studies on Th17/Treg cells, ROR- $\gamma$ t and Foxp3 in RA and other AIDs have been widely carried out abroad [4], but there are not many studies in China, and the results of the research are also inconsistent about the proportion of Th17 and (or) Treg cells in the pathogenesis of GD. The combined detection of Th17, Treg cells, ROR- $\gamma$ t, Foxp3, and the relationship with GD, is rarely reported. The etiology of AITD is complex, and its onset is not only related to environmental and immune factors, but also closely related to genes. The protein tyrosine phosphatase non-receptor 22 (PTPN22) is currently considered to be an important susceptibility gene related to autoimmune diseases [5], and its abnormal function can lead to rheumatoid arthritis [6], systemic lupus erythematosus [7], myasthenia gravis [8] and other autoimmune related diseases [9]. There have been many reports that single nucleotide polymerphism (SNP) of the PTPN22 gene is related to the above-mentioned diseases. AITD has similar genetic and immunological basis, but the specific mechanism of action has not been fully elucidated. Therefore, in-depth research on cellular immunity and genetic genetics is helpful to the diagnosis, treatment and prevention of AITD.

\section{Materials and Methods}

\subsection{Research Objects and Groups}

A total of 100 untreated inpatients with GD who were in the endocrinology department of Cangzhou Central Hospital and Cangzhou People's Hospital from September 2019 to August 2020 were selected as the experimental group. Among them, 24 were males and 76 were females, with an average age $(45.4 \pm 15.1)$. Sixty healthy people with matching gender and age at the same time were selected as the control group (NC group), including 16 males and 44 females, with an average age of $(44.8 \pm 16.9)$ years. There were no statistically significant differences in gender and age in each group. All patients met the diagnostic criteria of GD in the "Guidelines for the Diagnosis and Treatment of Thyroid Diseases of China" formulated by the Endocrinology Branch of the Chinese Medical Association in 2008 [10], and none of them had received Graves disease related treatment. According to the results of thyroid hormone examination and whether there are corresponding clinical symptoms or signs, they are divided into GD-A group (clinical hyperthyroidism: symptoms or signs of hyperthyroidism, FT3 $>7.1 \mathrm{pmol} / \mathrm{L}, \mathrm{FT} 4>22 \mathrm{pmol} / \mathrm{L}$, $\mathrm{TSH}<0.27 \mathrm{mU} / \mathrm{L}$ ) and GD-B group (subclinical hyperthyroidism: no symptoms or signs of hyperthyroidism, FT3 and FT4 are normal, $\mathrm{TSH}<0.27 \mathrm{mU} / \mathrm{L}$ ). Exclusion criteria: (1) Combin-ed with other autoimmune system diseases; (2) Acute and chronic infections, malignant tumors, pregnancy and allergic diseases; (3) Diabetes, abnormal liver function and blood system diseases; (4) Those who have used glucocorticoid therapy in the past 30 days. The evaluation and analysis of iodine nutritional status adopt the standards issued by the World Health Organization (WHO), the United Nations Children's Fund (UNICEF) and the International Council for the Control of Iodine Deficiency Disorders (ICCIDD) in 2007. Median urinary iodine (MUI) $<100 \mu \mathrm{g} / \mathrm{L}$ is iodine deficiency, $100-199 \mu \mathrm{g} / \mathrm{L}$ is adequate iodine, $200-299 \mu \mathrm{g} / \mathrm{L}$ is iodine excess, $\geq 300 \mu \mathrm{g} / \mathrm{L}$ is iodine over-dose [11].

This study was approved by the ethics committee, and all the study subjects signed an informed consent form.

\subsection{Research Methods and Projects}

\subsubsection{Specimen Collection and Processing}

(1) Specimen Collection: All research subjects fasted for more than 12 hours. Take $10 \sim 20 \mathrm{~mL}$ of fasting midsection urine and place it in a clean polyethylene plastic tube. Collect $5 \mathrm{~mL}$ of fasting heparin anticoagulated venous blood from 8:00 to 10:00 and store it in a refrigerator. (2) PBMC Separation: The Ficoll density gradient centrifugation method was used to separate peripheral blood mononuclear cells (PBMC), the PBMC concentration density of each sample was adjusted to $1 \times 10^{6} / \mathrm{L}$ in the incubation solution of RPMI1640 with FBS. Place the PBMC suspension in a 24-well culture plate, add $25 \mu \mathrm{g} / \mathrm{L}$ of phorbol ester (Alexis, USA) and $1 \mathrm{mg} / \mathrm{L}$ of inomycin (Alexis, USA) to each well, incubate in an incubator at $37^{\circ} \mathrm{C}$ for 4 hours, and collect cells. (3) RNA Extraction and cDNA Synthesis: Trizol method was used to extract total RNA from PBMC of HT patients and control groups. Use formaldehyde denaturing electrophoresis and absorbance value (A260/A280) to identify RNA quality. Take the RNA with the ratio of A260/A280 above 1.9 and at least 2 bands in electrophoresis for the following experiment, and adjust the RNA concentration of each sample to $0.5 \mu \mathrm{g} / \mathrm{L}$.

\subsubsection{Project Testing}

(1) Urinary Iodine Determination: Using the principle of iodine-catalyzed arsenic-cerium reaction, the urinary iodine content is detected by arsenic-cerium catalytic spectrophotometry (Conson Biochemicals, WS/T107-2016). The instrument is a fully automatic biochemical analyzer 
(Hitachi 7600-110). (2) Detection of the Ratio of Th17 and Treg Cells: Th17 cells were labeled with Th17 cell detection kit (FITC/CD3, PE/CD4, Alexa Fluor647/IL-17, Biolegend, USA), and Treg cells were labeled with Treg cell detection kit (FOXP3 Alexa Fluor 488/CD4 PE-Cy5/CD25PE) using Flow Cytometer (FACSC alibur, BD, USA). According to the instructions, add the antibody labeled with the corresponding fluorescent dye into the flow tube containing $100 \mu \mathrm{L}$ of blood sample, incubate at room temperature for $15 \mathrm{~min}$ in the dark, add the red blood cell lysate, mix and incubate at $4{ }^{\circ} \mathrm{C}$ for $10 \mathrm{~min}$ in the dark. After adding the PBS solution, mix and test on the machine. The test results are expressed as the percentage of positive cells and the fluorescence intensity of the positive cells, and the analysis data is obtained with Cell Quest software. (3) PCR and Genotyping: Trizol was used to extract total RNA from the sample, and cDNA was synthesized according to the reverse transcription procedure. PCR-SASP technology was used to amplify the PTPN22-1123 $\mathrm{G} / \mathrm{C}$ site. Design 3 primers with reference to the DNA sequence of PTPN22 in Gen Bank, including 1 common upstream primer (R) and 2 downstream primers (F1 and F2) for specific alleles at the 3'end, R: 5' ACCCTGCATATGTA-ATGCTGGT-3', 5'-CATTGAGAGGTTATGCAAGCTC-3', CATTGAGAGGTTATGCAAGCTG-3', F1 and R form a pair of primers to amplify the $\mathrm{G}$ allele, F2 and R form a pair of primers to amplify the $\mathrm{C}$ allele. The genotype is determined according to the presence or absence of the PCR product. PCR amplification was performed with $0.2 \mu \mathrm{g}$ DNA as a template. PCR procedure: $95^{\circ} \mathrm{C}, 5 \mathrm{~min} ; 40$ cycles $\left(95^{\circ} \mathrm{C}, 40 \mathrm{~s} ; 60^{\circ} \mathrm{C}, 40 \mathrm{~s}\right.$; $\left.72^{\circ} \mathrm{C}, 40 \mathrm{~s}\right) ; 72^{\circ} \mathrm{C}, 10 \mathrm{~min}$; The amplified products were electrophoresed on a $2 \%$ agarose gel, and genotype identifyca -tion was performed after staining. The PCR amplification product is purified and sequenced, and the target site peak map is analyzed according to the sequencing file to determine the genotype of the site.

\subsubsection{Statistical Methods}

Using SPSS 20.0 statistical software for statistical analysis, each group of genotypes and allele frequencies are tested for Hardy-Weinberg genetic balance to confirm whether they comply with the balance law, and to calculate the genotype frequencies and allele frequencies of each polymorphic locus. The $\chi 2$ test was used for comparison, and the odds ratio (OR) was used to express the relative risk, and $\mathrm{P}<0.05$ was considered as the difference was statistically significant.

\section{Results}

\subsection{SASP-PCR Analysis of PTPN22 Gene-1123}

The length of the amplified fragment of the PTPN22 promoter is $268 \mathrm{bp}$, the primer F1 is for allele G (wild type), and the primer $\mathrm{F} 2$ is for allele $\mathrm{C}$ (mutant type). When the genotype is GG, only the downstream primer F1 can amplify the PCR product; when the genotype is $\mathrm{CC}$, only the downstream primer F2 can amplify the PCR product; when the genotype is $\mathrm{GC}$, both downstream primers F1 and F2 can beamplify the PCR product. The results of this study detected three genotypes at the 1123 site (rs2488457) of the PTPN22 gene: $\mathrm{C} / \mathrm{C}, \mathrm{G} / \mathrm{G}, \mathrm{C} / \mathrm{G}$. Figure 1.

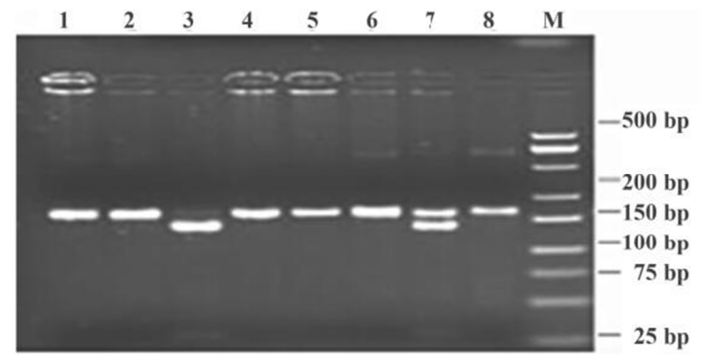

Figure 1. Results of PCR amplification with sequence specific primers at 1123 site in promoter region.

Note: Marker: 3. CC homozygote, 7. GG heterozygote, others are CG homozygote.

\subsection{Comparison of Genotype and Allele at 1123Site of PTPN22 Gene Between GD Patients and NC group}

The distribution of genotype and allele frequency at 1123 site of PTPN22 gene between GD patients and NC groupcon-formed to Hardy Weinbergprinciple, and the difference between the two groups was statistically significant $(\mathrm{P}<0.05)$. Table 1 .

Table 1. Comparison of genotype and allele frequency between GD patients and NC group [n (\%)].

\begin{tabular}{lllll}
\hline & GD group & NC group & $\chi 2$ & P \\
\hline Genotype & & & & \\
G/G & $29(29.0 \%)$ & $19(31.6 \%)$ & 5.429 & 0.047 \\
$\mathrm{C} / \mathrm{G}$ & $43(43.0 \%)$ & $28(46.7 \%)$ & & \\
$\mathrm{C} / \mathrm{C}$ & $28(28.0 \%)$ & $13(21.7 \%)$ & & \\
Allele & & & & 0.049 \\
$\mathrm{G}$ & $84(52.5 \%)$ & $81(50.6 \%)$ & 5.276 & \\
$\mathrm{C}$ & $76(47.5 \%)$ & $79(49.4 \%)$ & & \\
\hline
\end{tabular}

\subsection{Relative Risk of PTPN22 (rs2488457) Genotype and Allele for GD}

According to the generalized genetic model, calculatethe odds ratio (OR) value and $\mathrm{P}$ value of the genotype and the allele site (rs2488457) of the PTPN22 gene. OR value represents relative risk, $\mathrm{OR}=1$ means that the factor does not work, OR greater than 1 is a risk factor, OR less than 1 is a protective factor. The theoretical calculation formula of OR value: $\mathrm{OR}=(\mathrm{a} / \mathrm{b}) /(\mathrm{c} / \mathrm{d}) . \mathrm{P}<0.05$ is considered as statistically significant. The results showed that the $\mathrm{C} / \mathrm{G}$ and $\mathrm{C} / \mathrm{C}$ genotypes at $1123 \mathrm{G}>\mathrm{C}$ of PTPN22 gene can significantly increase the risk of $\mathrm{GD}$, and $\mathrm{G} / \mathrm{G}$ genotype can reduce the risk of GD. People with $\mathrm{C} / \mathrm{G}$ and $\mathrm{C} / \mathrm{C}$ genotypes are more likely to have GD disease (OR values are 1.762 and 1.406 respectively). $\mathrm{G} / \mathrm{G}$ genotype may be a protective factor for the occurrence of GD (OR value is 0.881 ). Table 2 . 


\section{Discussion}

Table 2. The relative risk of genotype and allele (rs2488457) of PTPN22 gene to $G D$.

\begin{tabular}{|c|c|c|c|c|c|}
\hline & \multirow{2}{*}{ OR } & \multicolumn{2}{|c|}{$95 \%$ CI } & \multirow{2}{*}{$\chi^{2}$} & \multirow{2}{*}{$\mathbf{P}$} \\
\hline & & Lower & Upper & & \\
\hline \multicolumn{6}{|c|}{ Genotype } \\
\hline $\mathrm{G} / \mathrm{G}$ & 0.881 & 0.240 & 1.763 & 13.127 & 0.062 \\
\hline $\mathrm{C} / \mathrm{G}$ & 1.762 & 0.381 & 1.341 & 10.204 & 0.041 \\
\hline $\mathrm{C} / \mathrm{C}$ & 1.406 & 0.252 & 1.186 & 11.431 & 0.048 \\
\hline \multicolumn{6}{|c|}{ Allele } \\
\hline G & 1.126 & -0.214 & 1.671 & 1.213 & 0.436 \\
\hline $\mathrm{C}$ & 1.027 & -0.386 & 1.438 & 1.186 & 0.619 \\
\hline
\end{tabular}

AITD is the disease with the highest incidence of organ-specific autoimmune diseases and the most common thyroid disease in humans, accounting for about $90 \%$ of thyroid diseases [12]. AITD mainly includes GD and HT. The former is also known as toxic diffuse goiter. It is a common clinical endocrine disease and the most common type of AITD It has the highest incidence of all types of hyperthyroidism, about $80 \%-86 \%$ Of hyperthyroidism is caused by GD. The clinical symptoms or signs of GD mainly include diffuse symmetrical goiter, hyperfunction, exophthalmos, and hypermetabolic state. The adverse effects of this disease on multiple systems can lead to multiple organ damage [13], the treatment effect is not good, and serious sequelae may occur. The incidence has rised in recent years. There are many causes of GD. A variety of factors such as environment, genetics and autoimmune dysfunction lead to specific autoimmune reactions in the thyroid gland, diffuse goiter, and hyperthyroidism [14]. Clinical studies have shown that there are many reasons for the increase in the incidence of GD, and iodine nutritional status may be one of the influencing factors. The effect of iodine on GD has received extensive clinical attention. In the 10 years before and after Universal Salt iodization (USI), with the increase of iodine nutrition level, the incidence of AITD increased. Studies have shown that with the increase in iodine intake, the incidence of GD and the positive rate of thyroid autoantibodies both increased. An increase in iodine intake can cause iodine-induced hyperthyroidism, which can change AITD from recessive to dominant or worse. Increasing iodine intake not only acts as an environmental stimulus, but also has a positive synergistic effect with susceptible HLA alleles in the occurrence and development of GD and HT to induce autoimmune processes. Liu Rong found that the external inspection rate and composition ratio of GD and HT showed a phased change trend with the change of iodine intake [15]. Recent studies have found that Th cells and Treg cells are involved in different types of immune responses and their cytokines and transcription factors play an important role in regulating the differentiation, proliferation and effector cytokine products of $\mathrm{T}$ cells $\left(\mathrm{CD}^{+}\right)$. IL-17, TGF- $\beta$, ROR- $\gamma \mathrm{t}$, and Foxp3 are the main cytokines and specific transcription factors secreted by Th17 and Treg cells respectively, they play an important role in immunosuppression. It also has become the current research direction of the mechanism of occurrence, development and outcome of immune-related diseases. In recent years, studies on Th17/Treg cells, ROR- $\gamma \mathrm{t}$ and Foxp3 in AID have been widely carried out abroad [4].

Some studies have shown that GD and HT have some common susceptibility genes and there may be familial aggregation [16]. Some studies have shown that GD and HT have some common susceptibility genes and may have family aggregation [16]. A large number of basic and clinical research data indicate that the onset of AITD is not only related to environmental and immune factors, but also closely related to genetics. Risk of GD patients' sibs is greater than $15 \%$ and $50 \%$ of AITDs patients have autoantibodies in their parents and siblings [17]. Research results show that about $80 \%$ of the susceptibility to GD is determined by genes [18]. Therefore, genetic susceptibility and environmental factors are closely related to AITDs and the study of genetic polymorphisms can better discover, diagnose and treat diseases, which has become one of the important directions of AITDs research. The protein tyrosine phosphatase nonreceptor 22 (PTPN22) is currently considered to be the most important human autoimmune disease susceptibility gene besides HLA and its abnormal function can lead to the occurrence of many autoimmune-related diseases $[5,7]$. At present, there have been many reports about the relationship between single nucleotide polymerphism (SNP) of PTPN22 gene and AID, but there are few studies on the correlation between Th17/Treg cytokines and PTPN22 gene -1123 site in GD patients with different iodine nutritional status. AITD has similar genetic and immunological basis, but the specific mechanism of action has not been fully elucidated. Therefore, in-depth research on cellular immunity and genetic genetics is helpful to the diagnosis, treatment and prevention of AITD.

It has been found that the susceptibility genes involved in the onset of GD are distributed on 16 chromosomes, including 30 genes in two major categories: immune regulation and thyroid intrinsic [19]. The PTPN22 gene is located on the short arm of human chromosome 1 (1p13.3-p13.1), which encodes a lymphoid protein tyrosine phosphatase (Lyp) composed of 807 amino acid residues with a relative molecular weight of approximately 110000 . Single nucleotide poly-merphism (SNPs) of PTPN22 gene mainly includes promoter-1123G $>\mathrm{C}$, exon $1858 \mathrm{C}>\mathrm{T}$ and intron (rs3789607). The protein expressed after mutation weakens or disappears to inhibit immune response, triggering AID. The Src family kinases (SFKs) is one of the members of protein tyrosine kinases. The Src family kinases (SFKs) is one of the members of protein tyrosine kinases. It mainly includes 9 family members. Among them, Src, Fyn and Yes are almost distributed through-hout the body, while other members such as Lck are exist in certain tissue cells. SFKs participate in the regulation of cell growth, differentiation and metabolism and have important significance in regulating cell signal transduction [20]. After $\mathrm{T}$ cell activation, Lyp forms a complex with C-terminal of Src tyrosine kinase (CSK kinase) through its SH3 domain normallyand causes Lck, Fyn and Zap-70 tyrosine kinases to deactivate in TCR signal transduction. That modulates $\mathrm{T}$ cell activity $[21,22]$. When the PTPN22 gene is mutated, its Lyp structure will change, it 
cannot form a complex with CSK kinase, which will change the $\mathrm{T}$ cell signal transduction pathway and ultimately lead to the occurrence and development of autoimmune diseases. As an important candidate gene for AID, if missense mutation of PTPN22 gene occurs at C1858T (rs2476601), it leads to the transformation of the 620 codon of LYP from arginine (Arg) to tryptophan (Trp), which makes LYP unable to bind to CSK, leading to weakened inhibition of $\mathrm{T}$ cell signal transduction and reduced TCR-mediated immune response [23]. PTPN22 participates in cellular immune response and is related to a variety of AIDs [24]. Smythreported that the variation $(1858 \mathrm{C}>\mathrm{T})$ of PTPN22 can increase the risk of type 1 diabetes (T1D) in 2004. It is a susceptibility gene for T1D inheritance. It was proved that the 1858 allele was associated with AITDs $[\mathrm{OR}=1.43(95 \%$ CI $1.17-1.76)]$ in 1734 cases of GD and the control group, which indicated that the PTPN-22 locus was associated with multiple AIDs [25]. The variation of $(1858 \mathrm{C}>\mathrm{T})$ of PTPN22 is closely related to the occurrence of multiple AIDs including AITD in the Caucasian population. In 2005, Vang found that Lyp-Trp620 is related to T1D, rheumatoid arthritis, GD and other AIDs [26].

Leereported that the overall odds ratios (ORS) for T-allele, $\mathrm{T} / \mathrm{T}$ and $\mathrm{T} / \mathrm{T}+\mathrm{C} / \mathrm{T}$ genotypes were significantly increased in RA, SLE, GD and T1D. That means (PTPN22) C1858T polymorphism is associated with autoimmune diseases [27].

But Chabchoub reported that the PTPN22 C1858T single nucleotide polymorphism has no or minor effect on RA and AITDs susceptibility in the Tunisian population [28]. Ban' study showed significant difference in the distribution of the haplotype suggests that the PTPN22 gene rather than rs2476601 is involved in the development of AITD in the Japanese population [29]. Early studies found that thePTPN22 gene $\mathrm{C} 1858 \mathrm{~T}$ (rs2476601) is closely related to the occurrence of T1D in Europeans. The PTPN22SNPsare closely related to the occurrence of a variety of AID including AITD in Caucasian population, but the SNP (rs2476601) has not been found in Asian and African population [30].

The susceptibility to GD depends on heredity, mainly genetic factors related to HLA and CTLA4 locus. In 1958, the UK reported for the first time that the T allele and genotype frequencies of PTPN22 (rs2476601) in 549 GD patients were significantly higher than those of healthy controls. Afterwards, studies on the relationship between PTPN22 C1858T gene polymorphism and autoimmune diseases such as GD have been successively carried out in many countries. However, the results of GD research in different countries and ethnic groups are not the same. Velaga reported that the PTPN22 1858T is the major susceptibility allele for GD in the British population [31]. Criswell studied 265 families from the Genetic Society of Multiple Autoimmune Diseases (MADGC) and found that PTPN22 1858T was significantly related to HT, but not to GD [32]. The PTPN22 1858T may not be a susceptibility allele for GD and the PTPN22 gene polymorphism in the Tunisian family has nothing to do with autoimmune thyroxine tyrosine phosphatase [33]. Analysis of the PTPN22 C1858T allele in 290 GD patients and 310 healthy controls in the Polish population revealed that the PTPN22 1858T allele is associated with GD. The median age of onset of GD patients: TT genotype (20.8 years old), CC genotype (42 years old), CT genotype ( 35 years old). the difference of median ageis more than twice. However, this gene polymorphism has no correlation with GD ophthalmopathy and goiter [34]. A total of 768 GD patients, 768 control subjects, and 313 families with autoimmune thyroid disease participated. The results showed the association of SNPs within the PTPN22 region differs between autoimmune diseases, occurring individually and/or as part of a haplotype, indicating that the mechanisms by which PTPN22 confers susceptibility to GD may, in part, be disease specific [35]. This indicates that the SNPs in the PTPN22 region have different connections between AIDs, occurring alone and/or as part of a haplotype, indicating that the mechanism by which PTPN22 confers susceptibility to GD may be partly diseasespecific. In 2008, Ichimura tested theG1123C polymorphism (rs2488457) inpromoter region, Arg620Trp (C1858T) polymorphism (rs2476601) in exon 14, IMS-JST146695 polymorphism (rs3789607) in intron 19 and SNP37 (rs3789604) downstream of the PTPN22 gene in GD patients $(n=414)$ and healthy subjects $(n=231)$. The results indicate that SNP37 of the PTPN22 gene is related to the susceptibility to GD in the Japanese population [36]. Zhebrun selected 170 GD patients with a disease course of less than 15 years and found that the frequency of the rs2476601 genotype was significantly higher than that of the control group $(\mathrm{p}<0.05$, $\mathrm{OR}=4.23$ ), and believed that the PTPN22-1858T allele may be the incidence of GD in Russia Susceptibility genes [37]. Luo conducted a Meta analysis of the relationship between 3764 AITDs and 3328 PTPN22 C1858T and GD susceptibility in 11 studies in PubMed, Embase, WanFang, and CNKI. It was found that there was statistical significance between the PTPN22 C1858T gene polymorphism and the risk of AITD,

PTPN22 C1858T is related to GD susceptibility in the UK, Russia, Poland, and Japan, but not related to HT, especially in whites [38]. López-Cano found that PTPN22 R620W was associated with GD susceptibility $(\mathrm{OR}=4.3, \mathrm{P}=0.004)$ in Mexico, but not with SLE susceptibility $(\mathrm{OR}=1.8, \mathrm{P}=0.19)$ [39]. Shehjar performed SNP (1858C/T) analysis on 135 GD and 150 healthy controls. The results showed that the detection rates of PTPN22 $1858 \mathrm{CC}, \mathrm{CT}$ and TT genotypes in GD patients were $97.7,2.2$ and $0 \%$, respectively. In the healthy control group, the detection rates of $\mathrm{CC}$ and $\mathrm{CT}$ genotypes were 100 and $0 \%$, respectively. There is no significant correlation between PTPN22 $1858 \mathrm{C} / \mathrm{T}$ and GD patients in Kashmir [40]. Some study suggested that PTPN22: 1858

$\mathrm{C}>\mathrm{T}$ gene polymorphism may be associated with a predisposition to GD within the adult north-eastern Polish population [41]. In Brazil, Bufalo and other researchers genotyped 282 GD patients and 308 healthy controls. The GG genotype of rs2476601 of PTPN22 gene was associated with positivity for both TgAb $(\mathrm{p}=0.0360)$ and TPOAb $(\mathrm{p}<0.0001)$. The CC genotype of rs3789607 of PTPN22 (OR=2.668, 95\% $\mathrm{CI}=1.399-5.086, \mathrm{P}=0.0029)$ is related to $\mathrm{GD}$ susceptibility [42].

PTPN22 polymorphism may affect the severity of the 
disease. Some researches on PTPN22 gene polymorphism and its relationship with AIDs have also being carried out in China. Zhang Zhihongused PCR-RFLP method to study the single nucleotide polymorphism of the exon14 of PTPN22 in 539 individuals. It was found that no homozygous TT genotype was detected, but the PTPN22 1858T allele frequency difference was statistically significant $(\mathrm{P}<0.05)$ [43]. Zhang tested the genotypes of PTPN22-C1858T of 1085 healthy populations and found that 31 of them were heterozygote (PTPN22-1858C/T), the frequency of PTPN22-1858T allele was $1.43 \%$ and the frequency of PTPN22-1858T was significantly different among 15 Chinese groups $(\mathrm{P}<0.01)$ [44]. Researchers conducted a genome-wide association study on 1536 cases of GD and 1516 cases of control group and found 255 common SNPs on LD block containing PTPN22 were associated with GD $(\mathrm{P}<0.05)$ [2]. Wang Shufound that the 1858 site of PTPN22 only has the $\mathrm{C}$ allele in the Han population in Shanghai, China, and has nothing to do with GD [45]. Ye Juan performed PTPN22 genotype analysis on 82 cases of GD and 70 cases of healthy people and found that the patients and healthy controls were all CC genotypes and no T allele was detected [46]. It suggested that the PTPN22 $\mathrm{C} 1858 \mathrm{~T}$ gene polymorphism has regional characteristics. Zheng Ruizhi analyzed the genotypes and allele frequencies at 1858 of the PTPN22 gene in 283 cases of GD patients and 218 normal people and found that the CC, CT, and TT genotypes in GD patients and the control group are $97.2 \%, 2.8 \%, 0 \%$ and $97.7 \%, 2.3 \%, 0 \%$, respectively. There was no statistically significant difference in genotype and allele frequency between the two groups. Homozygous TT genotype was not detected and $\mathrm{T}$ alleles were rarely detected [47]. Yu Zhiyun analyzed the PTPN22 gene $1858 \mathrm{C}>\mathrm{T}$ site, CTLA-4 gene $49 \mathrm{~A}>\mathrm{G}$ site and the PTPN22 gene $1123 \mathrm{G}$ in 149 cases of GD, 82 cases of HT and 131 healthy controls in Shanxi Province were analyzed. They found that there was no polymorphism at the $1858 \mathrm{C}>\mathrm{T}$ site of the PTPN22 gene and the alleles and genotype distribution frequencies of the PTPN22 gene $1123 \mathrm{G}>\mathrm{C}$ had statistically significant differences between the GD group and the normal control group [48].

Our study showed that the genotype of PTPN22 gene $1123 \mathrm{G}>\mathrm{C}(\mathrm{rs} 2488457)$ and the frequency distribution of $\mathrm{G}$ and $\mathrm{C}$ alleles in GD patients and the control group were signifiantly different (Table 1). The SNP (rs2488457) of PTPN22 gene is related to the occurrence of GD. People who carry the $\mathrm{G} / \mathrm{G}$ gene may reduce the risk of GD (OR value is 0.881). However, carrying $\mathrm{C} / \mathrm{G}$ and $\mathrm{C} / \mathrm{C}$ genotypes may be risk factors for the occurrence of GD (OR values are 1.762 and 1.406 , respectively). This is basically consistent with the research results reported by Yu Zhiyun [48], but inconsistent with the result of Ichimura $M$ [36]. We speculate that $1123 \mathrm{G}>\mathrm{C}$ is not the only mutation site in the PTPN22 gene which is associated with the onset of GD and polymorphisms at other sites also exist. Tizaoui [49] comprehensive meta-analysis on the relationship between PTPN $221858 \mathrm{C}>\mathrm{T}$ polymorphism

and autoimmune diseases. They found that PTPN22 is a candidate gene for a variety of autoimmune diseases. It may be a common mechanism of autoimmune diseases and has a huge impact on the understanding of autoimmunity. It may help formulate treatment and prevention strategies. Therefore, we should further study the pathogenesis of PTPN22 1858C/T and other polymorphisms in various autoimmune diseases.

The distribution of PTPN22 1858T varies greatly in different regions and populations around the world. The frequency of PTPN22 1858T is decreasing from north to south in Europe, such as $8 \%-10 \%$ in Western Europe and North America, up to $15 \%$ in Finland, while in Asia and Africa there is almost no T allele in healthy people [50]. PTPN22 C1858T is related to the occurrence of GD in the United Kingdom, Poland, Russia, and Brazil, but may not be related to the GD in Tunisia, Kashmir, Japan, and China. It may be related to the formation of differences in the genetic structure of populations in different regions, as well as the mutation history, migration, isolation, genetic drift and gene fusion of different populations. It may be related to the formation of differences in the genetic structure of populations in different regions, as well as the mutation history, migration, isolation, genetic drift and gene fusion of different populations. In addition to ethnic and geographic factors, the susceptibility genes of GD may also be closely related to factors such as life history and environment. Whether it is related to other haploids or mutations in a single susceptibility gene, the sample size should be further expanded and further verified in different regions and ethnic groups.

At present, research on environmental, immune and genetic factors in AITD has been carried out, it has been found that excess iodine is positively correlated with thyroid diseases. High iodine intake can affect the secretion of thyroid hormones and the level of autoantibodies in GD patients.

The thyroid function, autoantibodies, Th17/Treg cell ratio and function, and corresponding cytokines and transcription factors of GD patients under different iodine nutrition conditions have changed accordingly, and they participate in the occurrence and development of GD. Our team found that the higher the urine iodine level, the more obvious the changes in thyroid hormone, the higher the level of autoantibodies, the more severe the damage to thyroid function [51]. Our research results also showed that the higher the urine iodine level, the more obvious the changes in thyroid hormones and the higher the level of autoantibodies. It shows that high iodine may be the main environmental factor that induces the occurrence and development of GD. The proportion of Th17 cells, serum IL-17 and ROR- $\gamma$ t in the PBMC of GD patients were significantly increased, while the proportion of Treg cells and Foxp3 mRNA expression were significantly reduced, and the serum TGF- $\beta$ was reduced. The ratio of Th17/Treg cells in GD patients was significantly positively correlated with TPOAb and $\mathrm{TgAb}$ titers. TPOAb and $\mathrm{TgAb}$ antibody titers are highly correlated with Th17/Treg, IL-17 and ROR- $\gamma \mathrm{t}$ [52]. It shows that Th17/Treg cell immune imbalance and the changes of factors are involved in the immune damage of thyroid tissue itself. GD disease may have abnormalities in urinary iodine levels, thyroid function, TPOAb, TgAb, Th17/Treg cells and related factors. This study found that there was no statistically 
significant difference between the $\mathrm{G} / \mathrm{G}, \mathrm{C} / \mathrm{G}, \mathrm{C} / \mathrm{C}$ genotypes and the $\mathrm{G}$ and $\mathrm{C}$ gene frequencies at the PTPN22 gene $1123 \mathrm{G}>\mathrm{C}$ site (rs2488457) between GD patients and the control group under different iodine nutrition conditions, It shows that the iodine nutritional status and the $1123 \mathrm{G}>\mathrm{C}$ polymorphism of PTPN22 gene have no correlation with the onset of GD. But it still needs to be confirmed by a large-sample prospective study.

\section{Conclusion}

Through the study of PTPN22 gene polymorphism and Th17/Treg cells and their factors with different iodine nutritional status in GD patients. We found that the proportion of Th17 cells, serum IL-17 and ROR- $\gamma \mathrm{t}$ in the PBMC of GD patients with different iodine nutritional status were significantly increased, while the proportion of Treg cells and Foxp3mRNA expression were significantly decreased, serum TGF- $\beta$ decreased. The ratio of Th17/Treg cells in GD patients was significantly positively correlated with the titers of TPOAb and TgAb and the titers of $\mathrm{TPOAb}$ and $\mathrm{TgAb}$ antibodies were significantly correlated with Th17/Treg, IL-17 and ROR- $\gamma$ t. There is a certain correlation between Th17 and Treg cells and factors in PBMC of GD patients and PTPN22 gene 1123G $>$ C locus (rs2488457). The distribution frequencies of various genotypes and alleles have statistically significant differences between GD and the control group ( $\mathrm{P}<0.05$ ), The polymorphism ( $\mathrm{rs} 2488457$ ) is related to GD susceptibility, and further research should be done to clarify the mechanism of PTPN22 gene1123G $>$ C in the pathogenesis of GD. From the level of cellular immunity and molecular genetics, it provides a basic basis for the early diagnosis of GD, precise treatment and exploration of the mechanism of cellular immunity and genetic genetics of the disease, and opens up new ways for scientific prevention.

\section{Acknowledgements}

Cangzhou Key R \& D Plan and Guidance Project (Project No: 1833020011).

Natural Science Foundation of Cangzhou Medical College (No. 18Z015).

\section{References}

[1] Chen X, Mei Y, He B, et al. General and specific genetic polymorphism of cytokines-related gene in AITD [J]. MediatorsInflamm, 2017, 2017 (1): 3916395.

[2] Xue L, Pan C, Gu Z, et al. Genetic heterogeneity of susceptibility gene in different ethnic popu-lations: refining association study of PTPN22 for Graves'disease in a Chinese Han population [J]. PLoS One. 2013, 8 (12): e84514.

[3] Su JP, Su SO, Zhang B, et al. The effects of different a mounts of iodine in take on the immunestatus of patients with Graves disease [J]. Clin Med China, 2012, 28 (1): 44-46.

[4] Hou ZJ, Mu ZX, Wang CC. Research Progress of Th17/Treg Cells and Their Transcription Factors in Autoimmune Diseases
[J]. American Journal of Clinical and Experimental Medicine 2019, 7 (4): 83-92.

[5] Tang QL, Xue YM. Hashimoto's thyroiditis related gene research progress [J]. Drug Research, 2013, 10 (15): 42-46.

[6] Chen MH, Dai B. Relationship between protein tyrosine phosphatase nonreceptor type 22 and rheumatoid arthritis [J]. Foreign Medicals Ciences Ectionof Medgeography, 2014, 35 (3): $252-255$.

[7] Li Sheng Dong. Analysis of association between PTPN22 and transcription factor FOXP3 gene polymorphisms and genetic susceptibility to systemic lupus erythematosus [J]. Chin Mode Doct, 2017, 55 (5): 1-4.

[8] Su Y. Association Study between PTPN22 Gene Polymorphism and Myasthenia Gravis [D]. Master's thesis of shandong university, 2019.

[9] $\mathrm{Xu} \mathrm{QH}, \mathrm{Wu}$ J, Chen ZT. Advances in the Research of the SPNs of PTPN22 Gene and Autoimm-UneRelated Diseaes [J]. Med J Wuhan Unive, 2017, 38 (5): 844-849.

[10] Chinese Medical Association Endocrine Credits "China thyroid disease treatment guidelines" Writing Group. Chinese Guidelines for the Diagnosis and Treatment of thyroid diseases-Hyperthy-roidism [J]. Chin J Intern Med, 2007, 46 (10): 876-882.

[11] Chinese Medical Association Endocrine Credits "China thyroid disease treatment guidelines" Writing Group. China thyroid disease treatment guidelines-iodine defi ciency disorders $[\mathrm{J}]$. Chin J Intern Med, 2008, 47 (8): 689-690.

[12] Chen Xiaoheng, Mei Yizhou, He Bei, et al. General and Specific Genetic Polymorphism of Cyto-kines-Related Gene in AITD Mediators Inflamm. 2017, 2017: 3916395.

[13] Wang L, Wang B, Chen SR, et al. Effect of Selenium Supplementation on Recurrent Hyperthyroidism Caused by Graves' Disease: A Prospective Pilot Study [J]. Horm Metab Res, 2016, 48 (9): 559-564.

[14] Li HX, Xiang N, Hu WK, et al. Relation between therapy options for Graves' disease and the course of Graves' ophthalmopathy: a systematic review and meta-analysis $[\mathrm{J}]$. J Endocrinol Invest, 2016, 39 (11): 1225-1233.

[15] Liu R. Twenty-eight year analysis of diffuse toxic GOITER, Hashimoto's thyroiditis, and pathological study of Vegf, tgf, CD20, CD45RO expression in diffuse toxic goiter, Hashimoto's thyroiditis [D]. Tianjin Medical University, Master's thesis, 2007.

[16] Muhali FS, Song RH, Wang X, et a1. Genetic variants of BANK1 gene in autoimmune thyroid diseases: a case-control association study [J]. Exp Clin Endoerinol Diabetes, 2013, 121 (9): 556-560.

[17] Teng XL, Zhang XM. Advances in Single-nucleotide polymer-phism and autoimmune thyroid disease [J]. J Bengbu Med Coll, 2013, 38 (11): 1519-1522.

[18] Zhang YD, Tan LN, Wei HY, et al. Meta-analysis of CTLA-4 promoter-49 A/Gpolymorphism and Graves'disease in China [J]. Chin J Endocr Surg, 2013, 7 (1): 64-68.

[19] Wang SQ, Yao YL. Research progress on toxic diffuse goiter and PTPN-22 gene polymorphism [J]. Front Med, 2016, 6 (3): $8-10$. 
[20] Zeng QJ, Chen HY, Su JP. Src family kinases affect the expression of Nav1.1 in spiral ganglion neurons [J]. J Clin Otorhinolaryngol Head Neck Surg (China), 2014, 28 (11): 789-792.

[21] Chen XH, Mei YZ, He B, et al. General and Specific Genetic Polymorphism of Cytokines-Related Gene in AITD [J]. Mediators Inflamm. 2017, 2017 (1): 3916395.

[22] Kleinewietfeld M, Hafler DA. The plasticity of human Treg and Th17 cells and its role in autoimmunity [J]. Semin Immunol. 2013, 25 (4): 305-312.

[23] Lempainen J, Laine AP, Hammais A, et al. Non-HLA gene effectson the disease process of type 1 diabetes: From HLA susceptibilityto overt disease [J]. J Autoimmun, 2015, 61: 45-53.

[24] Wang L, Zhang L, Xiao $\mathrm{H}$, et al. Interference of protein tyrosine phosphatase non-receptor type 22 inhibits NLRP3 inflammasome activation to alleviate inflammation after cerebral hemorrhage in rats [J]. J Third Milit Med Univ, 2019, 41 (24): 2409-2416.

[25] Smyth D, Cooper JD, Collins JE, et al. Replication of an association between the lymphoid tyrosine phosphatase locus (LYP/PTPN22) with type 1 diabetes, and evidence for its role as a general autoimmunity locus [J]. Diabetes.2004, 53 (11): 3020-3023.

[26] Vang T, Congia M, Macis MD, et al. Autoimmune-associated lymphoid tyrosine phosphatase is a gain-of-function variant [J]. Nat Genet.2005, 37 (12): 1317-1319.

[27] Lee YH, Rho YH, Choi SJ, et a1. The PTPN22 C1858T functional polymorphism andautoimmune diseases--a meta-analysis Rheumatol, 2007, 46 (1): 49-56.

[28] Chabchoub G, Teixiera EP, Maalej A, et al. The R620W polymorphism of the protein tyrosine phosphatase 22 gene in autoimmune thyroid diseases and rheumatoid arthritis in the Tunisian population [J]. Ann Hum Biol, 2009; 36 (3): 342-349.

[29] Ban Y, Tozaki T, Taniyama M, et al. Association of the protein tyrosine phosphatasenonreceptor 22 haplotypes with autoimmune thyroid disease in the Japanese population $[\mathrm{J}]$. Thyroid, 2010, 20 (8): 893-899. Thyroid.2010, 20 (8): 893-899.

[30] Song HD. Recent progress in identificafion of candidate genes of Graves' disease [J]. Clin J Endocrinol Metab, 2011, 27 (12): 961-966.

[31] Velaga MR, Wilson V, Jennings CE, et al. The codon 620 tryptophan allele of the lymphoidtyrosine phosphatase (LYP) gene is a major determinant of Graves' disease [J]. J Clin Endocrinol Metab. 2004, 89 (11): 5862-5865.

[32] Criswell LA, Pfeiffer KA, Lum RF, et al. Analysis of families in the multiple autoimmune disease genetics consortium (MADGC) collection: the PTPN22 620W allele associates with multiple autoimmune phenotypes [J] Am J Hum Genet. 2005, 76 (4): 561-571.

[33] Chabchoub G, Maalej A, Petit-Teixeira E et al. Polymorphisms in the protein tyrosine phosphatase (PTPN22) gene is not associated with autoimmune thyroid in a large affected Tunisian family [J]. Clin Immunol.2006, 120 (2): 235-236.

[34] Skórka A, Bednarczuk T, Bar-Andziak E, et al. Lymphoid tyrosine phosphatase (PTPN22/LYP) variant and Graves' disease in a Polish population: association and gene dose-dependent correla-tion with age of onset [J]. Clin Endocrinol (Oxf).2005, 62 (6): 679-682.

[35] Heward JM, Brand OJ, Barrett JC, et al. Association of PTPN22 haplotypes with Graves' disease [J]. J Clin Endocrinol Metab. 2007, 92 (2): 685-690.

[36] Ichimura M, Kaku H, Fukutani T, et al. Associations of protein tyrosine phosphatase nonreceptor 22 (PTPN22) gene polymorphisms with susceptibility to Graves' disease in a Japanese population [J]. Thyroid. 2008, 18 (6): 625-630.

[37] Zhebrun D, Kudryashova Y, Babenko A, et al. Association of PTPN22 1858T/T genotype with type 1 diabetes, Graves' disease but not with rheumatoid arthritis in Russian population [J]. Aging (Albany NY).2011, 3 (4): 368-673.

[38] Luo L, Cai B, Liu F, et al. Association of protein tyrosine phosphatase nonreceptor 22 (PTPN22) C1858T gene polymorphism with susceptibility to autoimmune thyroid diseases: a metaanalysis [J]. Endocr J, 2012, 59 (5): 439-445.

[39] López-Cano DJ, Cadena-Sandoval D, Beltrán-Ramírez O, et al. The PTPN22 R263Q polymorphism confers protection against systemic lupus erythematosus and rheumatoid arthritis, while PTPN22 R620W confers susceptibility to Graves' disease in a Mexican population [J]. Inflamm Res. 2017, 66 (9): 775-781.

[40] Shehjar F, Dil-Afroze, Misgar, Riaz A, et al. PTPN22 1858 C/T Exon Polymorphism is not Associated with Graves' Disease in Kashmiri population [J]. Indian J Endocrinol Metab.2018, 22 (4): 457-460.

[41] Wawrusiewicz-Kurylonek N, Koper-Lenkiewicz OM, Gościk J, et al. Association of PTPN22 polymorphism and its correlation with Graves' disease susceptibility in Polish adult population-A preliminary study [J]. Mol Genet Genomic Med. 2019, 7 (6): e661.

[42] Bufalo NE, Dos Santos EB, Rocha AG, et al. Polymorphisms of the genes CTLA4, PTPN22, CD40, and PPARG and their roles in Graves' disease: susceptibility and clinical features [J]. Endocrine. 2021, 71 (1): 104-112.

[43] Zhang ZH, Zhang XL, Chen F, et al. Genetic polymorphism of rheumatoid arthritis related gene PTPN22 C1858T in 6 ethnic groups in China [J]. Inter J Genetics, 2007, 30 (2): 81-84.

[44] Zhang ZH, Chen F, Zhang XL, et al. PTPN22 allele polym orphism $\mathrm{s}$ in 15 Chinese populations [J]. Int J Immunogenet, 2008, 35 (6): 433-437.

[45] Wang S, Zhao ZF, Jiang XH, et al. Relationship between LYP /PTPN22 and Graves' disease in Han nationality from Shanghai Of China [J]. J Shanghai Jiaotong Univ (Medical Science), 2007, 27 (8): 984-986.

[46] Ye Juan, Ren An. Relationship between PTPN22 gene polymorphism andGraves' diseasein Han nationality from Anhui province [J]. China Journal of Modern Medicine, 2015, 25 (7): 44-46.

[47] Zheng RZ, Zhang SH, Li R, et al. Study on the relationship between polymorphism of PTPN22 and Graves 'disease of Han nationality in Chongqing area $[\mathrm{J}]$. J Chongqing Med Univ, 2008, 33 (1): 22-24.

[48] Yu ZY, Zhang JA, Maier-haba, et al. Association of polymorphism of protein tyrosine phosphatasenonreceptor-22 gene with AITD [J]. Chin J Cell Mol Immuno, 2008, 24 (8): 804-807. 
[49] Tizaoui K, Kim SH, Jeong GH, et al. Association of PTPN22 1858C/T Polymorphism with Autoimmune Diseases: A Systematic Review and Bayesian Approach [J]. J Clin Med, 2019, 8 (3), 347 (1-22).

[50] Ban Y, Tozaki T, Taniyama M, et al. The codon 620 single nucleotide polymorphism of the protein tyrosine phosphatase22 gene does not contribute to autoimmune thyroid disease suscep-tibility in theJapanese [J]. Thyroid, 2005, 15 (10): 1115-1118.
[51] Jing Feng, Cuicui Wang, Zhenjiang Hou, et al. Correlation Analysis of Thyroid Function and Autoantibodies in Graves' Disease Patients with different Iodine nutritional Status [J]. Science J Public Health, 2020, 8 (6): 168-176.

[52] Jing Feng, Cuicui Wang, Zhaoxin $\mathrm{Mu}$, et al. Research on the Role of Th17Treg Cells and their Factors in Graves' Disease with different Iodine Nutritional Status [J]. Science J Public Health, 2021, 9 (1): 1-11. 